\title{
Analysis on the Emotional Design of Children's Medicine Packaging
}

\author{
Hu Kang, Zhang Ruike \\ Wuhan University Science and Technology
}

\begin{abstract}
In order to improve the design of children's medicine packaging and help children overcome the fear of medicine, this paper analyzes the psychological needs of consumers, and introduces the idea of "people-oriented" emotional design into the packaging design. From three levels of instinct, this paper analyzes the application, behavior and reflection of emotional design in children's medicine packaging. Finally, it discusses the emotional design of children's medicine packaging in terms of graphics, color, modeling and interactive design. It is concluded that children can get emotional care and psychological comfort through packaging. Emotionalization is the future development trend of children's medicine packaging.
\end{abstract}

Keywords—Emotional design; Children's medicine packaging; People-oriented; Interactive design

\section{INTRODUCTION}

As a kind of special commodity, medicine must give people a sense of security while clearly communicating the information on its packaging. As children's medicine packaging for special populations, more attention needs to be paid. Children's medicine packaging requires both basic information and emotional interaction with patients. This requires designers to focus on the users for better understanding the psychological and emotional needs of the users.

\section{ANALYSIS OF CONSUMERS' PSYCHOLOGICAL NEEDS}

Emotional design is particularly important for the analysis of consumers' psychological needs. Only by understanding the emotional needs of users can we solve problems through emotional design. As a kind of special commodity, children's medicine must face both direct users and parents who are the decisive factors in purchasing. Therefore, users should be analyzed from both users and consumers.

\section{A. Children's Medicine Consumption Psychology}

1) Selection Psychology

When children are unwell, the parents will be anxious to go to the pharmacy to buy medicine, usually go directly to the children's area to find the right medicine. The main factors for parents to get the information and decide to buy medicine include common knowledge of medicine, consultation with doctors, recommendation from shop assistants, introduction by relatives and friends, and advertising media. Parents are anxious when their children are sick. When it comes to a wide variety of medicines packaged in similar packages, the choice is difficult. Parents pay attention to the choice of medicine information without excessive reading. In the case of the same product quality, brand, production date and other factors, they are more willing to accept the medicine packaging that children like.

\section{2) Use Psychology}

In order to make the children recover as soon as possible, the parents will give the children medicine immediately after purchase. Before taking it, it's essential to first understand the ingredients and functions of the medicine, especially the method of use, dosage and precautions, and the safety of the user. Therefore, it is particularly important to display clear information, design clear patterns and match reasonable color on the medicine packaging, which can provide more help to parents. As consumers' spending power increases, they pay more attention to spiritual satisfaction and pleasure in the process of consumption, and they want a sense of comfort that "good medicine is no longer bitter."

\section{B. Consumers' Psychology for Children's Medicine}

1) Consumption Psychology of Children

Children's nature is lively and active, and they are curious about everything. They dare to try anything. They have strong curiosity, like imitation, and are easily affected by the external environment. As they grow up, children's emotions are gradually improved, mainly in the sense of responsibility, honor, aesthetics and other emotions. The ability to control emotions is also strengthened. Their self-awareness develops rapidly and they are good at expressing themselves. However, their independence, attention and other willpower are weak.

\section{2) Consumption Psychology of Adults}

With the development of society, families pay more and more attention to children and respect children's choices, but children are still in the stage of immature development, so parents still play a leading role in consumption. Children's difficulty in taking medicine has also become a problem for parents, so when choosing medicine, parents will choose products that children are not resistant to. There are also fixed consumption patterns for adults when purchasing products. They are more inclined to the texture and beauty of packaging with function and safety than flat and boring packaging. Innovative packaging and individual design and color are conducive to the brand's sales and promotion. 


\section{APPLICATION OF EMOTIONAL DESIGN IN CHILDREN'S MEDICINE PACKAGING}

The so-called emotional design "is to incorporate emotions into the design works through various shapes, colors, textures and other modeling elements, to inspire people's associations in the process of consumers' appreciation and use of products, to resonate and obtain spiritual pleasure and emotional satistaction". From the perspective of cognitive psychology, Norman proposed the "emotional design hierarchy model", which divided the user's cognitive processing into the instinct, behavior and reflection.

\section{A. Design of Instinct Level - Design of Shape}

The instinct level is the basis of the emotional processing. It is mainly reflected in the color, graphics and shape of packaging in children's medicine packaging design. These are the most direct design elements to mobilize children's emotions. The instinct level involves the most primitive physiological characteristics. Children make a first impression on the products through sight, touch and smell, which causes the inner emotional fluctuation to produce emotional experience. The emotionalization of form can be designed through the prototype of nature, plants and animals so as to vividly satisfy children's innocence and pursuit of fun. Color plays an extremely important role in children's understanding of nature, society and selves. In the design, it should be in line with children's aesthetic and color perceptions. Different colors presented on the packaging will bring different emotional experiences to children. Therefore, in the design of medicine packaging, emotional consideration should be fully considered and the role of color in psychology should be exerted. The designer meets the design requirements of the instinctive level in the design of the medicine packaging. Transforming visual language into a way to spread emotions breaks children's resistance and fear, and brings happiness to children through interesting packaging.

\section{B. Design of Behavioral Level - Extension of Function}

The emotional behavioral level is mainly reflected in children's medicine packaging. Children get pleasant emotional experience in the process of participation, use and operation. This requires designers to understand users and solve problems through design, starting from their needs and difficulties in use. In the packaging of children's medicine, designers can design an additional function of knowledge and ingenuity, so that children can achieve the effects of edifying sentiment, increasing knowledge, and exercising ability when using the products, thus achieving an emotional care design.

\section{Design of Reflection Level -- Satisfaction of Inner Emotion}

The reflection level is the total impression and reflection of the products after long-term use by consumers. A familiar feeling can give users a sense of trust and emotional resonance. In the design of children's medicine packaging, children's cognition should be grasped. By incorporating the familiar shapes and memories of children into the packaging design, familiar emotional memories will give children a sense of security. By grasping the children's behavioral and emotional characteristics, the purpose of satisfying the needs of the inner emotional level is realized on the basis of the instinct level and behavior level.

The emotional reflection level involves the cultural level. China's culture is profound, so it has a wealth of design elements that can be applied to medicine packaging. It can not only meet the needs of medicine packaging, but also allow children to contact traditional Chinese culture and love the culture of the motherland from an early age.

\section{EMOTIONAL DESIGN OF CHILDREN'S MEDICINE PACKAGING}

\section{A. Affinity Design of Images}

In order to eliminate children's fear for medicine, the affinity design of children's medicine is particularly important. "Studies have shown that when consumers get outside information, $83 \%$ of their impressions come from sight, $11 \%$ from hearing, $3.5 \%$ from smell, and $1.5 \%$ from touch." The visual experience brought by the image has an important emotional promotion effect.

At present, there are some improvements in the packaging of children's medicine on the market. The use of cartoon patterns has become a common design method. The cartoon image is very important in the hearts of children. The cartoon image represents innocence. The cartoon characters of medicine packaging on the market can be roughly divided into two categories. The first category is the characters that children are familiar with from cartoons. The Bread Superman series of children's cough syrup is the image of the bread superman in the popular Japanese cartoon, which represents bravery and justice, which also encourages the children who take the medicine to fight against the difficulties with courage (Figure 3 ). The second category is cartoon images recreated based on the characteristics of medicine itself.

All affinity images are designed to capture the children's psychology and convey positive emotions to the children. This can help them build confidence and identity, and give them a sense of affinity and warmth, which can greatly alleviate the aversion to medicine. Children's observations are more delicate, so good packaging design should be reflected in some details, because the emotional care design in the details is more touching.

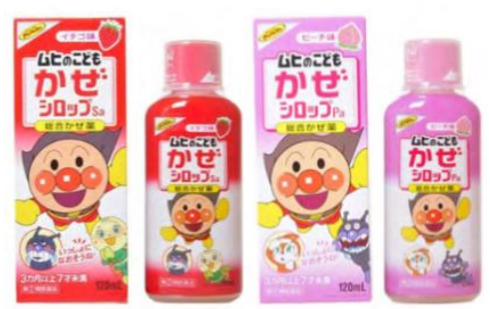

Fig. 1 The Bread Superman Series of Children's Cough Syrup 


\section{B. Associative Application of Color}

As the most active factor in visual communication, color is very important in medicine packaging. For example, antiinflammatory, hypothermia and calming medicine packages are designed in blue and green, making people feel cool and quiet. The use of color factors to convey product information to consumers is an indirect concern. But children, as a special group, are rich in imagination and color-sensitivity. They like the color of fruits, the color of seasons, and the natural color. Different color bands will give them different feelings. It is not suitable for large-area use of white or black on the packaging of medicine, which will bring discomfort and dislike to children. And boys prefer colder color, girls prefer warmer color, etc., and the special characteristics of children should be considered in design.

\section{Interesting Conveyance of Shapes}

Interesting shapes for children can be seen as their toys, which can close the distance between children and medicine. The interesting design can bring comfort to children, attract children's attention while bringing happiness to them, and effectively solve the problem of parents' difficulty in feeding medicine. The medicine packaging of a flower shape changes the way the original tablets are arranged, so that the tablets are arranged in the shape of flowers, and the plastic sheet in the shape of a flower pot at the end of the tablet can be opened into a holder, like a pot of flowers placed on a table. When the tablets are eaten, the plastic behind them will lift up like blooming flowers, symbolizing a positive attitude, which will encourage the sick children and give them an unexpected surprise (Figure 5).

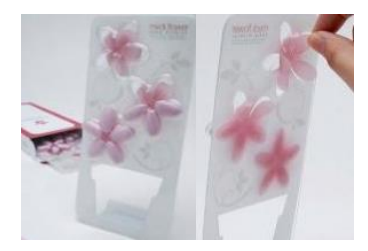

Fig. 2 The medicine Packaging of a Flower Shape

\section{Interactive Experience Communication}

Emotional interaction design is a new development trend in user-centered modern design. It aims at user emotion and embodies a kind of humanistic care. The interactive packaging design establishes a good communication bridge between the packaging and the consumers, enabling consumers to participate in the packaging and communicate the packaging information to achieve a deep sense of pleasure. The interaction is applied to the packaging of children's medicine, so that the children can feel the comfort and psychological satisfaction while experiencing the emotional interaction, which has a good promotion effect on the acceptance of medicine and the rehabilitation of the bodies and minds.

There are two main methods of emotional interaction design. The first is to stimulate the human sense through the visual image, which leads to emotional resonance. This method is based on the visual perception theory of Gestalt psychology. "Gestalt psychology is based on "shape" and breeds people's psychology and emotions in "shape". "Things and art forms have the nature of human emotions." The other method of emotional interaction design is to adopt humanized design, "adhere to the principle of human-centered, comprehensively consider the physiological structure, psychological conditions, behavioral habits and other factors in the design process, and then determine the design orientation".

\section{CONCLUSION}

Emotional children's medicine packaging still has a lot of space to study and practice. This article explores the analysis of children's medicine packaging and the emotional design methods through the three emotional levels. With images, color, shapes and interactive design as the foothold, the care design of children's medicine packaging is carried out to eliminate children's fear and rejection and to help children's medicine packaging improvement. Children's products should be more in line with children's emotional cognition, which reflects the care and respect for this special group of children. Emotional children's medicine packaging will become a trend and reflect the value of packaging design.

\section{REFERENCES}

[1] Xu Yan. Medicine Packaging Design Based on Patients' Psychological Needs [J]. Packaging Engineering, 2008, (8): 139-141.

[2] Su Jianning, Li Heqi. Method of Product Form Design Based on Perceptual Image $[\mathrm{J}]$. Chinese Journal of Mechanical Engineering,2004,40(4) : 164-167.

[3] Kong Weiyan, Sun Wenxiang. Application Analysis of Interactive Concept in Packaging Design [J]. Packaging Engineering, 2016, 37(10): $35-38$.

[4] Ni Qian, Jiang Lu. Emotional Design of Drug Packaging[J]. Packaging Engineering, 2014, 35(4): 13-16

[5] Zhu Hui, Huang Zhigang, Zhang Yudong. Care About Vulnerable Groups and Secure ---Packaging Design of Children 's Drugs [J]. Packaging Engineering, 2006, (4): 157-165.

[6] Tang Sha. Research on Medicine Packaging Design Based on Alleviating Bad Emotions - Taking Children's OTC Medicine Packaging as an Example. Master's Degree Thesis of Hunan University of Technology,2014.

[7] Catharine Fishel. Children's Product Design Strategy [M]. Shanghai: Shanghai People's Fine Arts Publishing House,2003.

[8] Xu Na. Research on the Use of Cartoon Characters in Children's Product Packaging [D]. Shanghai: East China Normal University, 2010. 